\section{Die grosse Demontage}

\author{
E. Taverna
}

Die «Progress Foundation» nennt sich ideologisch unabhängig und entwickelt unsere Zivilisation, indem sie "die fördernden und hemmenden Umstände ermittelt». Ihre "liberale Idee» fusst auf "Wettbewerb, Privateigentum, Selbstverantwortung und soziale Verantwortung". Die Stiftung finanziert Stipendien und Forschungsprojekte und organisiert jährliche Konferenzen und Seminare. 1995 zur Rationierung im Gesundheitswesen, in diesem Jahr in Schwarzenberg, im spätherbstlichen Bregenzerwald, zum Thema "Privatsphäre und Geheimhaltung".

Das dicke Dossier besteht aus Buchkapiteln zweier amerikanischer Autoren. Von Charles J. Sykes, einem Moralapostel der republikanischen Rechten, «The End of Privacy", und von Richard W. Rahn, Chefökonom der USA Handelskammer, "The End of Money and the Struggle for Financial Privacy».

\section{Der erste Tag}

Die einzige eingeladene Frau hat kurzfristig abgesagt. Es verbleiben die restlichen vierzehn Männer beim Apéro. Bankleute und Lehrstuhlinhaber stossen an, die Economiesuisse, ehemals Vorort, das Volkswirtschaftsdepartement und der Ethikphilosoph. Sogar ein Pater und ein Mediziner trinken mit. Der kühle Weisswein fördert die Stimmung, lockert die Vorstellrituale und den einsetzenden Smalltalk. Der Kursleiter von der NZZ stellt sich als Anhänger des Friedrich August von Hayek vor und begrüsst seine Gäste zum "sokratischen Gespräch", das keine Verpflichtungen beinhalte, aber doch da und dort einen Bericht auslösen möge. Das leckere Abendessen und das gemütliche Zimmer erzeugen eine erwartungsvolle Neugier.

\section{Der zweite Tag}

Das erste Gespräch ist ein Versuch, die Privatsphäre zu umschreiben. Der vorliegende Text verkürzt diese auf "the right to be let alone" oder umschreibt das Private als Zone intimer Beziehungen, wo Dampf ablassen, sich erholen und gehen lassen unbeobachtet möglich sind. Dieser abgeschlossene Bereich und das demokratische, öffentliche Leben bedingen sich gegenseitig. Alle sind sich einig, dass die neuen Informationstechnologien dieses verletzliche Gleichgewicht zunehmend bedrohen. Obwohl das persönliche Eigentum nur eines von vielen menschlichen Grundrechten ist, werden sich die folgenden Gespräche auf Besitz und Vermögen konzentrieren. Geld und Kapital sind die einzigen Triebkräfte des Fortschritts.
Noch lässt sich der eigene Körper nicht kaufen, und alles, was sein Innenleben betrifft, soll Unbefugte nichts angehen. Medizinische Informationen sind besonders sensibel und im elektronischen Verkehr gefährdet, vor allem wenn zentrale Datenbanken diese für «managed care»-Zwecke vernetzen. Der anwesende Datenexperte zitiert den Gesetzgeber, der einzelne und Institutionen wirksamer schützt als in den USA. Trotzdem sind es nicht nur unlöschbare Harddisks und Hacker, die Kryptogramme und "firewalls" durchlöchern, auch Konsumentenprofile, Datenverkauf, Nachlässigkeit und gut gemeinte, aber überflüssige Erhebungen bringen Kunden und Bürger in Gefahr. Das Bankgeheimnis scheint besonders schützenswert. Selbst das Patientengeheimnis ist kein Vorbild mehr, weil es zu viele Ausnahmen kennt. Da bleibt als Vorbild nur noch das Beichtgeheimnis. So radikal wie Sykes, der dem Staat den totalen Datenkrieg gegen alle Bürger und Bürgerinnen unterstellt, will sich niemand äussern. Doch staatliche Monopole sind prinzipiell schlecht, weil jede Regulierung Knappheit erzeugt. Ein Teilnehmer sieht im illegalen Organhandel die Folge staatlichen Eingreifens. Eine Niere aus Indien wäre ohne falsche Moral ein ganz gewöhnliches Tauschgeschäft zum gegenseitigen Nutzen. Die Vor- und Nachteile der Videoüberwachung öffentlicher Räume sind umstritten. Der Arbeitgeber darf damit seine Angestellten überwachen, denn er ist der Hausherr. In der U-Bahn oder vor der Abfallsammelstelle gilt das Prinzip der vom Stimmbürger auszuhandelnden Verhältnismässigkeit.

Dem Muskateller folgen der Zander und das Tischgespräch. Der Manchesterliberalismus war ganz anders und viel besser. Etiketten wie neo-liberal sind nicht beliebt. Liberal, ordo-liberal oder libertär klingt besser, auch wenn die Begriffe diffus bleiben. Doch Staatsinterventionen sind schädlich, wenn sie nicht das Kapital schützen und nur Vermögensbesitz garantiert die Freiheit. Auch ist soziale Gerechtigkeit nicht definierbar. Gesellschaftsvertrag? - nie gehört. Darwins "Survival of the fittest" gerät zum Leistungsausweis für nie zu hoch bezahlte Spitzenmanager. Intelligenz ist genetisch lokalisierbar. Die Misere Afrikas wird daraus verständlich.

\section{Der dritte Tag}

Der Diskussionsleiter entwirft seine unternehmerische Utopie von der Abschaffung des Staates. Ihn freut die Behauptung von Sykes, dass die Behörden das Rennen im Cyberland bereits verloren haben. Dank dem globalen Datenfluss in privaten Händen werde es keine Kriege mehr geben. Die Vision bleibt nicht ohne Widerspruch von der Wirtschaftsseite. Die folgende Steuerdebatte füllt den ganzen Vormittag. Leider kann das Ausland unserer subtilen Unterscheidung von Steuerhinterziehung und Steuerbetrug nicht folgen. Für einmal wird die Hegemonie des amerikanischen Rechtssystems bedauert. Am 
schlimmsten wütet der Staat, wenn er Einkommen und Vermögen besteuert. Denn diese Zwangsabgaben sind Leistungen ohne Gegenleistung, sie werden suboptimal oder gar nicht investiert. "Ab dreissig Prozent beginnt der Totalitarismus.» Steuererleichterungen sind für alle gut. Das belegen die USA, wo es allen immer besser geht. Nur dank den Reichsten überleben die Ärmsten der Welt. Der Sozialstaat fördert nur Begehrlichkeiten aller Art. Haarsträubende Beispiele werden aufgezählt, wie das vom Fürsorgeempfänger, der beim deutschen Staat prozessrechtlich einen teureren Schulranzen durchsetzte, weil das Kind sonst in der Schule ausgelacht werde. Überhaupt sollten abschreckende Beispiele von Fürsorgefällen publik gemacht werden, denn wer zahlt hat ein Recht auf Transparenz. Rechtshilfe in Steuersachen sind konsequent $\mathrm{zu}$ verweigern, schliesslich hat jeder Mensch gute Gründe, irgendwo auf der Welt sein Geld zu verstecken. Die Schweiz sollte ihre Standortvorteile nutzen, statt diese in vorauseilendem Gehorsam preiszugeben. Leider muss nachgeben, wer im ameri- kanischen Markt mithalten will, wo jüdische Kreise mit der Clinton-Administration zusammenarbeiten. "Wir gelangen hier an politische Abgründe».

Ein Buch macht die Runde, falls alle liberalen Stricke reissen. "How to be invisible" von J. J. Luna. Zum Inhalt gehören nützliche Tips zu «hidden ownership, bank accounts and money transfer".

Wieder klingen die Gläser im schönen Wälder Barockhaus. Das Rassismusgesetz ist so unnatürlich wie "political correctness". Beides schafft erst die Probleme, die es zu bekämpfen vorgibt. Wie das so gewesen sei mit dem freien Markt in Pinochets Chile? Bedauerlich zwar, aber unverzichtbar zur langfristigen ökonomischen Gesundung. Und schlimm, wie Haiders Privatleben von der bösen Jelinek mit Dreck beworfen wurde, wo auch das mit seinem Vermögen doch gar nicht stimmt.

Wie leicht wäre der Fortschritt zu haben, wenn die Regulierungskommissare nicht wären, die Gewerkschaften, die Feministinnen und Linken. Doch wenigstens sind hier alle furchtbar nett. 